\title{
Minimum entransy dissipation principle for optimization of transport networks
}

\author{
X.B. Liu ${ }^{1}$, M. Wang ${ }^{2,3 *}$, J. Meng', E. Ben-Naim ${ }^{3}$, Z.Y. Guo ${ }^{1}$ \\ ${ }^{1}$ Department of Engineering Mechanics, Tsinghua University, Beijing 100084, China \\ ${ }^{2}$ Earth and Environmental Sciences Division, Los Alamos National Laboratory, Los Alamos, \\ NM 87545, USA \\ ${ }^{3}$ Center of Nonlinear Study, Los Alamos National Laboratory, Los Alamos, NM 87545, USA
}

\begin{abstract}
The concept of entransy, which describes the capability of heat conduction in the continuum and was originally used for optimization of heat transfer devices, has been extended for optimization of transport networks in this contribution. Based on the definition of the entransy dissipation rate for transport networks, the analysis indicates that the minimum entransy dissipation rate leads to the optimal transfer performance of transport network subject to a given constraint. The present optimization analyses agree well with the existing experimental data and optimization theories for transport networks.
\end{abstract}

Keywords: transport network optimization; entransy dissipation rate; Murray’s law

\section{Introduction}

Transport networks extensively exist in the natural world and the human life. For examples, the subground water and the leaf venations of trees in nature, the windpipe network in lungs and the blood vessel network in human bodies, and the water, gas, oil and power supplies of a city or even a country. The optimization of transport networks has gained much more increasing attentions in recent years due to its importance though challenging [1-7]. It has been widely accepted that the mechanical/electrical energy dissipation rate is minimized if a hydraulic/electrical network is optimal [3-5,8-11]. Ordonez [7] studied the optimal structure of flow network which connected one point to a number of points by minimizing the fluid power losses, and Durand [3,4] obtained the optimal flow networks in terms of minimizing the mechanical dissipative energy with respect to two constraints: certain total channel volume and certain total channel surface area. Bohn [5] introduced an electrical energy dissipation rate function, which should be minimized for an optimal electrical transport network. Rodriguez [10] explained the tree-like structure and some empirical relationships of the river drainage network by the principles of minimum energy expenditure.

However, the minimum energy dissipation principle is hardly applicable directly to heat and mass transportation networks, because the concept of energy is unsuitable (for a mass transport network) or conserved (for the thermal energy in a heat conduction network) rather than dissipated. New principles had to be developed for the optimization of heat and mass transfer processes. A constructal theory has been proposed to construct an optimal heat conduction network for efficient cooling of a heat-generating volume [11] by minimizing the overall temperature difference of the network, where the energy dissipation principle

\footnotetext{
${ }^{*}$ Corresponding author. Email: mwang@lanl.gov
} 
is not applicable as the thermal energy conserves.

Most transport processes can be treated as movement of a generalized "mass" driven by some "potential difference". Thus people can abstract some common characteristics among various transport networks such as electrical, hydraulic, and heat or mass transport networks. For example, the analogy between a thermal system and an electric system is apparent, and it is easy to construct an electric network that would behave exactly like a heat conduction network system. Therefore, once the constructed electric network was optimized with the minimum energy dissipation principle [5] , the corresponding heat conduction network may be optimized as well. Alternatively, one can obtain the structure of optimal heat conduction networks by identifying and minimizing the physical quantity of a thermal system which corresponds to the concept of energy in electric/hydraulic systems.

Recently, a physical quantity, "Entransy", was proposed by Guo et al. [12] to characterize the heat transfer capability of an object. In analogy with the theory system of electricity or mechanics, the entransy of an object, defined as $U T / 2$, is featured as the "potential energy" of the internal energy $U$ at the temperature $T$. Though the thermal energy is conserved, the entransy dissipates in heat transfer processes and the entransy dissipation rate can be used as a criterion for optimization of heat transfer. Examples have shown successes of minimum entransy dissipation rate principle for optimization of heat and mass transfer in complex systems [13-15].

Inspired by Guo's theory [12] , this paper will try to extend the concept of entransy into heat and mass transport networks. The concept of entransy dissipation rate in heat/mass transport network is comparable to the concept of energy dissipation rate in electrical [5] and hydraulic transport networks [3,4,9-11]. Analysis using the minimum entransy dissipation principle for optimizations of heat and mass transport networks will be compared with the existing theoretical and experimental data.

\section{Entransy and entransy dissipation rate}

Based on the entransy concept [12] , the heat transfer capability of a system can be characterized by the entransy $(G)$ of the system, calculated as a half of the integral of the product between the internal energy $(U)$ and the thermal potential $(T)$ :

$G=\int_{\text {system }}\left(\frac{1}{2} U T\right)_{\Omega} d \Omega$

where $\Omega$ is the normalized volume of the system. The specific entransy is therefore defined as:

$g=u T / 2$

with $u$ representing the specific internal energy, calculated by

$u=\rho c T$

where $\rho$ is the density and $c$ the specific heat capacity.

For heat conduction without a heat source in an object, the thermal energy conservation equation is:

$\rho c \frac{\partial T}{\partial t}=-\nabla \cdot \vec{q}$

where $q$ represents the heat flux.

Equation (4) multiplied by the temperature yields the entransy balance equation:

$\rho c T \frac{\partial T}{\partial t}=-\nabla \cdot(T \vec{q})+\vec{q} \cdot \nabla T$

Based on the definition of specific entransy, Eq. (2), the entransy balance equation can then be rewritten as:

$\frac{\mathrm{d} g}{\mathrm{~d} t}=-\nabla \cdot(\vec{q} T)-J$

where $J=-\vec{q} \cdot \nabla T$ and it has a physical meaning as follows. Eq. (6) describes the time variation law of specific entransy. The first term on the right side is the entransy transfer rate accompanying the heat transfer process, and the second term could be 
understood as the entransy generation or dissipation. Since the heat flux ( $\vec{q}$ ) vector direction is always opposite against that of the temperature gradient $(\nabla T)$, we define $J$ as the entransy dissipation rate per unit volume in the heat transfer process. Eq. (6) indicates that, unlike the thermal energy, the entransy is not conserved during heat transfer processes.

The entransy dissipation rate per unit volume, which measures the heat transfer irreversibility, is proportional to the square of local temperature gradient as:

$$
J=-\vec{q} \cdot \nabla T=k(\nabla T)^{2}
$$

Especially, for a one-dimensional steady thermal conduction channel with a constant thermal conductivity, the entransy dissipation rate in the whole channel can be calculated by the entransy flow difference between inlet and outlet [12], which is proportional to the squared temperature difference and inversely proportional to the heat transfer distance:

$$
J_{\text {channel }}=Q T_{\text {in }}-Q T_{\text {out }}=k A \frac{\left(T_{\text {out }}-T_{\text {in }}\right)^{2}}{d}
$$

where $T_{\text {in, }} T_{\text {out }}, d, Q=q A, K=k A$ are the inlet and outlet temperatures, the characteristic length of the channel, the heat flow and the channel thermal conductance with A being the channel cross sectional area, respectively.

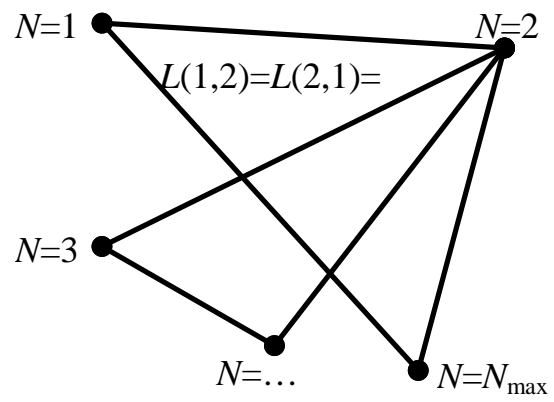

Fig. 1 General model of transport network

For an $N$-node heat conduction network as shown in Fig. 1, suppose the whole network composing of many one-dimensional thermal conduction channels described by Eq. (9). Each node may have $\mathrm{N}-1$ such channels with the other nodes. The channel conductance between the i-th node and the $\mathrm{j}$-th node, $K_{i, j}$, is treated as zero if there is no really channel between them. The entransy dissipation rate for the whole network can be calculated based on the Eq.(8):

$$
J_{\text {network }}=\sum_{i, j} K_{i, j} \frac{\left(\Delta T_{i, j}\right)^{2}}{d_{i, j}}
$$

where $\Delta T_{i, j}$ is the temperature difference and $d_{i, j}$ the characteristic length of channel from the $i$-th to the $j$-th node.

The entransy dissipation rate for the whole network can be expressed in terms of difference between input entransy ( from heat source) and output entransy flow ( from heat sink) at nodes as:

$J_{\text {network }}=\sum_{i, j} Q(i, j) \cdot \Delta T(i, j)=\sum_{n} T_{n} S_{n}$

where $T_{n}$ is the temperature and $S_{n}$ the heat source for the n-th node.

Based on the similarity between the heat transfer and the diffusive transport, the entransy and its dissipation concepts can be extended to the diffusion process. The entransy dissipation rate for the diffusion driven by concentration gradient can thus be defined as:

$J^{\text {mass }}=D(\nabla C)^{2}$

where $D$ and $C$ represent the diffusion coefficient and concentration. Through a very similar process of Eq. (9), the entransy dissipation rate for a steady mass diffusion network is expressed as:

$J_{\text {network }}^{\text {mass }}=\sum_{i, j} \mathrm{D}_{i, j} \frac{\left(\Delta C_{i, j}\right)^{2}}{d_{i, j}}$

For a network with mass sources, the entransy dissipation rate of the system can be expressed as:

$J_{\text {network }}^{\text {mass }}=\sum_{i, j} Q_{i, j}^{\text {mass }} \Delta C_{i, j}=\sum_{n} C_{n} S_{n}^{\text {mass }}$ 
where $Q_{i, j}^{\text {mass }}$ represents the mass flow through a channel $(i, j)$ from the $i$-th to the $j$-th node, and $S_{n}^{\text {mass }}$ the mass source on the n-th node.

\section{Minimum entransy dissipation principle}

Let us consider the physical significance of the minimized entransy dissipation rate. Suppose the symbol $F$ representing the generalized flux, $S$ the generalized source and $P$ the generalized potential for either heat or mass transfer. For a steady system, the sum of all heat/mass sources is zero. When at least one source does not equal zero, we can define the sum of all positive sources as the injecting heat/mass flux $F^{*}$ :

$$
F^{*}=\sum_{n:\left\{S_{n}>0\right\}} S_{n}=\sum_{n:\left\{S_{n}<0\right\}}-S_{n}
$$

Thus the entransy dissipation rate in Eq. (11) or (14) can be rewritten as:

$$
\begin{aligned}
J_{\text {network }} & =F^{*}\left(\sum_{n:\left\{S_{n}>0\right\}} \frac{S_{n}}{F^{*}} P_{n}-\sum_{n:\left\{S_{n}<0\right\}} \frac{-S_{n}}{F^{*}} P_{n}\right) \\
& =F^{*} \overline{\Delta P}
\end{aligned}
$$

Eq. (15) indicates that the entransy dissipation rate $J$ is determined by the injecting flow $F^{*}$ and the weighted average potential difference $\overline{\Delta P}$. For a given injecting flow $F^{*}$, a minimized entransy dissipation rate leads to a minimum average potential difference in the system. In other words, for example of a heat transfer network, we can minimize the driving weighted average temperature difference by minimizing the entransy dissipation rate at fixed injecting heat flux, which means the heat transport network is optimal. The mass transport network is very similar.

\section{Optimization Applications}

This section gives two examples that demonstrate the applications of minimum entrasy dissipation rate principle for the optimization of heat and mass transport networks. Three steps are performed for the optimizations: i) write out the entransy dissipation rate for the system based on the analysis in Section II; ii) find out the correct expression of constraint for optimization; iii) build the optimization function and do the optimization. The present demonstrations and theoretical analysis provide new explanations of optimal thermal conduction structure and biological hydraulic network structure, and will be compared with the existing theories and experimental data as well.

\section{A. Volume-point problem}

Cooling enhancement using high-conductivity material structures has very popular and important applications in N/MEMS device designs, such as CPU or micro fuel cells [14]. Fig. 2 shows a typical structure of a high-thermal-conductivity material distributed in a volume with uniform heat source. The volume needs to be cooled through a small boundary patch (point) located in the left center of the volume. The problem is to optimize the distribution of the network material in the volume for the best cooling effect, i.e. the highest temperature in the volume is minimized subject to the given internal heat source. This problem was often referred to as the volume-point problem [11]. A T-structure network solution of volume-point problem is demonstrated using the constructal theory, as shown in Fig. 2, where the high thermal conductivity material was distributed as hierarchical interconnected channels with different widths, and a parent channel was split perpendicularly into two equal daughter channels.

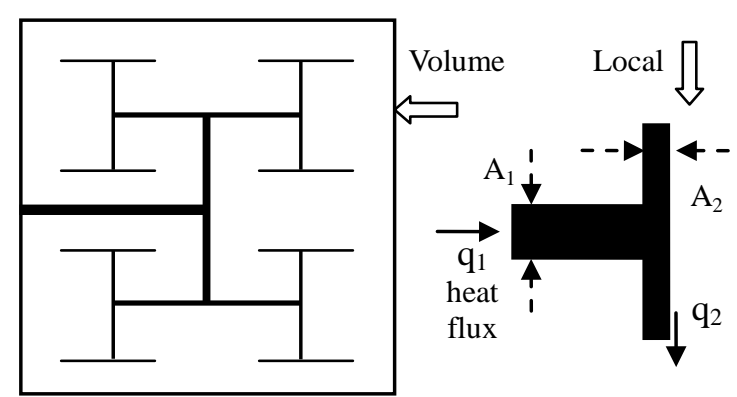

Fig. 2 Volume-point problem 
Assuming the background thermal conductivity is much smaller than that of the network material, we can regard that the thermal transport occurs mainly through the T-structure network. The Fourier's conduction law gives the heat flow for each channel as:

$Q_{i}=k A_{i} \frac{\Delta T_{i}}{L_{i}}$

where $A_{i}$ and $L_{i}$ are the cross-sectional area and length of the $i$-th channel, respectively.

Based on Eq. (8), the entransy dissipation rate is

$$
J_{\text {network }}=\sum_{i} k A_{i} \frac{\left(\Delta T_{i}\right)^{2}}{L_{i}}
$$

The constraint is the given total volume of the high thermal conductivity material. Therefore we build the optimization function by introducing a Lagrange multiplier $\lambda$ to minimize the entransy dissipation rate [5] :

$$
\Pi_{\text {heat }}=J_{\text {network }}-\lambda \sum_{i} V_{i}=\sum_{i} k A_{i} \frac{\left(\Delta T_{i}\right)^{2}}{L_{i}}-\lambda \sum_{i} A_{i} L_{i}
$$

In order to minimize the entransy dissipation rate, the partial derivative of the Lagrange function $\Pi_{\text {heat }}$ with respect to $A_{\mathrm{i}}$ has to be zero:

$$
\frac{\partial \Pi_{\text {heat }}}{\partial A_{i}}=\sum_{i}\left[k \frac{\left(\Delta T_{i}\right)^{2}}{L_{i}}-\lambda L_{i}\right]=0
$$

A generalized solution for Eq. (19) is $\Delta T_{i}=\alpha L_{i}$, where $\alpha=\sqrt{\lambda / k}$ is a constant. The conservation of heat at a junction gives $Q_{1}=2 Q_{2}$, which leads to the relation between the cross-sectional area of a parent channel and that of the daughter channels:

$A_{1}=2 A_{2}$

This result agrees well with the optimization design using the constructal theory, which concluded $A_{i} / A_{i+1} \rightarrow 2$ for $i \geq 3$ [11].

\section{B. The optimal vasculature of mammalian animals}

As well known, the vasculature system of mammalian animals consists of multi-level blood vessel networks, as shown in Fig. 3. The blood flow in the hydraulic network is driven by the heart power of the animal, and nutrients were transported to the tissue throughout the body. Since the pumping power of the heart of an adult animal is approximately a constant, there must be an optimal blood vessel network where the transmural nutrients transfer rate is maximized. We can further affirm that the current mammalian animal vasculature structure has maximized the transmural nutrients transfer rate during the long period of evolution and natural selection.

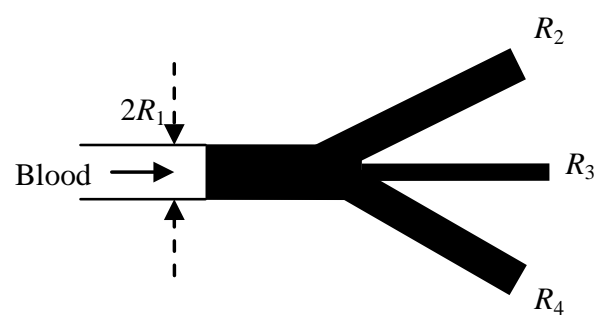

Fig. 3 A junction of blood vessels

The transmural nutrients transport from blood to the tissue is a mass transfer process. The "driving force" is the concentration difference $\Delta C$, and the mass flux per unit blood vessel surface area $F_{\text {mass }}$ is proportional to $\Delta C$ according to a linear transport law, $F_{\text {mass }}=D_{m} \Delta C$, where $D_{m}$ is the mass diffusion coefficient.

Eq. (12) gives the entransy dissipation rate for one-dimensional diffusive process. For the transmural nutrients transport in blood vessel network, the entransy dissipation rate for the $i$-th vessel is proportional to the vessel surface area as well [13] :

$J_{i}^{\text {mass }}=2 \pi r_{i} d_{i} \cdot D_{m}(\Delta C)^{2}$

with assuming the blood vessels are straight tubes with a radius of $r_{i}$ and a length of $d_{i}$. Therefore the total entransy dissipation rate is: 


$$
J_{\text {network }}^{\text {mass }}=\sum_{i} J_{i}^{\text {mass }}=D_{m}(\Delta C)^{2} \cdot \sum_{i} 2 \pi r_{i} d_{i}
$$

The constraint is the given heart pumping power. Suppose the blood flow in the vessels obeys the Hagen-Poiseuille's law. The heart pumping power $W$ equals to the total viscous dissipation rate of all blood vessels:

$$
W=\sum_{i} \frac{\pi r_{i}^{4}}{8 \mu} \frac{\left(\Delta p_{i}\right)^{2}}{d_{i}}
$$

where $\mu$ is the viscosity of the blood and $\Delta p_{i}$ the pressure drop within the length $d_{i}$.

The optimization function is:

$\Pi_{\text {mass }}=J_{\text {nelwork }}^{\text {mass }}-\lambda W=D_{m}(\Delta C)^{2} \sum_{i} 2 \pi r_{i} d_{i}-\lambda \sum_{i} \frac{\pi r_{i}^{4}}{8 \mu} \frac{\left(\Delta p_{i}\right)^{2}}{d_{i}}$

The partial derivative of $\Pi_{\text {mass }}$ with respect to radius $r_{i}$ have to equal zero to maximum the vessel surface area and therefore the nutrients transfer performance of the hydraulic network under the constraint of Eq. (23):

$$
\frac{\partial \Pi_{\text {mass }}}{\partial r_{i}}=0
$$

A generalized solution of Eq. (25) is $\Delta p_{i}=\beta d_{i} / r_{i}^{3 / 2} \quad$,where $\quad \beta=\sqrt{4 \mu D_{m} \Delta C / \lambda}$ approximated to be a constant. The mass at the junction (in Fig. 3) conserves when the nutrients mass transfer through the vessel surface is negligible in contrast to the mass flowing in the vessel $m_{1}=\sum^{n} m_{i}$.

The Hagẻn-Poiseuille's law results in:

$$
\frac{\pi r_{1}^{4}}{8 \mu} \frac{\Delta p_{1}}{d_{1}}=\sum_{i=2}^{n} \frac{\pi r_{i}^{4}}{8 \mu} \frac{\Delta p_{i}}{d_{i}},
$$

which leads to:

$$
r_{1}^{2.5} \propto \sum_{i=2}^{n} r_{i}^{2.5}
$$

Sherman [17] measured the vessel radii and distributions of the blood vessel of the small intestine of dogs and compared the data with the
Murray' law. In this work, we re-calculated S

Sherman's data of the first 4 ranks using different power numbers and listed the results in Table I. The results show that the summation increases with the level remarkably for a power number at 2 and decrease greatly for that at 3 . The relative standard derivation of summations of radii to the 2.5th power is significantly lower than those of the other two exponents calculated in Table 1, which strongly supports the present optimal radius relation of Eq.(27). This result suggests that the vasculature system of mammalian animals is an optimal hydraulic transport network which has the

\begin{tabular}{|c|c|c|c|c|c|}
\hline Rank & $\begin{array}{l}\text { Radius } \\
\mathrm{R}(\mathrm{m})\end{array}$ & $\mathrm{N}$ & $\sum R^{2}$ & $\sum R^{3}$ & $\sum R^{2.5}$ \\
\hline 0 & $1500 \times 10^{-6}$ & 1 & $2.250 \times 10^{-6}$ & $3.375 \times 10^{-9}$ & $8.714 \times 10^{-8}$ \\
\hline 1 & $500 \times 10^{-6}$ & 15 & $3.750 \times 10^{-6}$ & $1.875 \times 10^{-9}$ & $8.385 \times 10^{-8}$ \\
\hline 2 & $300 \times 10^{-6}$ & 45 & $4.050 \times 10^{-6}$ & $1.215 \times 10^{-9}$ & $7.015 \times 10^{-8}$ \\
\hline \multirow{2}{*}{3} & $40 \times 10^{-6}$ & 1440 & \multirow{2}{*}{$6.534 \times 10^{-6}$} & \multirow{2}{*}{$0.498 \times 10^{-9}$} & \multirow{2}{*}{$5.602 \times 10^{-8}$} \\
\hline & $96 \times 10^{-6}$ & 459 & & & \\
\hline \multirow{2}{*}{4} & $25 \times 10^{-6}$ & 8640 & \multirow{2}{*}{$18.04 \times 10^{-6}$} & \multirow{2}{*}{$0.470 \times 10^{-9}$} & \multirow{2}{*}{$9.207 \times 10^{-8}$} \\
\hline & $26.5 \times 10^{-6}$ & 18000 & & & \\
\hline \multicolumn{3}{|c|}{$\begin{array}{l}\text { Relative standard } \\
\text { deviation }\end{array}$} & $92.4 \%$ & $81.0 \%$ & $18.8 \%$ \\
\hline
\end{tabular}
best transmural nutrients transport performance subject to the constraint of a given total pumping power.

Table I Vessel data from small intestine of dogs

It is also noticed that the optimal radius relationship in Eq.(27) is similar to the famous Murray's law but with a different exponent. As well known, the Murray's law has been validated in many cases, such as the water transport in plants [1] and river network on the earth [9]. The former studies derived the Murray's law based on the minimum mechanical energy dissipation and the constraint of total blood vessel volume [16,17]. Based on the minimum entransy dissipation principle, we can also get the Murray's law in Eq. (28) if the optimization objective is to maximize the flow rate through the network subject to a given total network volume. 
$r_{1}^{3} \propto \sum_{i=2}^{n} r_{i}^{3}$

This result indicates that the optimized transport network structure depends on the optimization objective and the constraint. If the optimization objective is to maximize the diffusive transport flux through the network surfaces subject to a given pumping power, such as the bronchia network in lung and the vasculature system in intestine, the optimal network structure obeys the law of Eq. (27); however, if the optimization objective is to maximize the flow rate through the network subject to the constraint of a given total network volume, such the artery system in animals or water transport system in plants, the optimized network structure obeys the Murray's law of Eq. (28).

\section{Conclusions}

The concept of entransy has been extended for the optimization of heat and mass transport networks. Based on a definition of the entransy dissipation rate for heat and mass transport networks, the entransy analysis indicates that the minimum entransy dissipation rate leads to the optimal transport performance of heat and mass transport network subject to certain constraints. The minimum entransy dissipation principle has been validated by comparing with the existing experimental data and other optimization theories for different transport networks. The entransy analysis gets good agreements with the constructal theory for optimal thermal conduction network. The optimal structure of mass transfer network depends on the optimization objective and the constraint. When the optimization objective is to maximize the diffusive transport flux through the network surfaces subject to a given pumping power, for example of the vessel networks in small intestine, the optimization result indicates that the radii for different levels satisfy the $2.5^{\text {th }}$ power law, which has better agreements with the existing anatomical data of the small intestine of dogs than the classical Murray's law. If the optimization objective is to maximize the flow rate through the network subject to the constraint of a given total network volume, the optimized network structure obeys the Murray's law.

\section{Acknowledgements}

This work is supported by the Major State Basic Research Development Program of China (No.2007CB206901).

\section{Reference}

[1] McCulloh KA, Sperry JS, Adler FR. 2003 Water transport in plants obeys Murray's law. Nature. 421 939-942

[2] Colizza V, Banavar JR, Maritan A, Rinaldo A. 2004 Network structures from selection principles Phys. Rev. Lett. 92198701

[3] Durand M. 2006 Architecture of optimal transport networks. Phys. Rev. E. 73016116

[4] Durand M. 2007 Structure of optimal transport networks subject to a global constraint. Phys. Rev. Lett. 98088701

[5] Bohn S, Magnasco M O. 2007 Structure, scaling, and phase transition in the optimal transport network. Phys. Rev. Lett., 98088702

[6] Barber RW, Emerson DR. 2008 Optimal design of microfluidic networks using biologically inspired principles. Microfluidics Nanofluidics. 4 179-191

[7] Ordonez J C, Bejan A, Cherry R S. 2003 Designed porous media: Optimally nonuniform flow structures connecting one point with more points. Int. J. Therm. Sci., 42 857-870 
[8] Klarbring, A. and J. Petersson, et al. 2003 Topology optimization of flow networks. Comp. Meth. Appl. Mech. Eng. 192 3909-3932

[9] Dodds, P. S. and Rothman, D. H. 1999 Unified view of scaling laws for river networks Phys. Rev. E, 59 4865-4877

[10] Rodriguez-Iturabe, A Rinaldo, 1997 Fractal River Basisns: Change and Self-Organization, Cambridge

[11] Bejan A. Constructal-theory network of conducting paths for cooling a heat generating volume. Int. J. Heat Mass Transf. 1997, 40(4): 799-816

[12] Guo ZY, Zhu HY, Liang XG. 2007 Entransy--A physical quantity describing heat transfer ability. Int. J. Heat Mass Transf., 50 2545-2556

[13] Wei S, Chen L, Sun F. 2008 “Volume-point” heat conduction constructal optimization with entransy dissipation minimization objective based on rectangular element. Sci. China Ser. E: Tech. Sci., 51 1283-1295

[14] Wang CY. 2004 Fundamental models for fuel cell engineering. Chem. Rev. 104 4727-4765

[15] Chen Q, Wang M, Pan N, and Guo ZY. Irreversibility of heat conduction in complex multiphase systems and its application to the effective thermal conductivity of porous media. International Journal of Non-linear Science and Numerical Simulations 10 (1): 57-66, 2009

[16] Murray CD. 1926 The Physiological Principle of Minimum Work. I. The Vascular System and the Cost of Blood Volume. Proc. Nat. Acad. Sci. USA., 12 207-214

[17] Sherman TF. 1981 On connecting large vessels to small. The meaning of Murray's law. J. Gen. Physiol., 78 431-453 